\title{
Antimicrobial Susceptibility Profile of Bacteria in Mastitis Cow Milk Samples
}

\author{
George Cosmin NADĂȘ, Cristiana Ștefania NOVAC*, Cosmina Maria BOUARI, Ioana Adriana \\ MATTEI and Nicodim Iosif FIȚ
}

Faculty of Veterinary Medicine, University of Agricultural Sciences and Veterinary Medicine, 3-5 Calea Mănăștur st., Cluj-Napoca, Cluj, 400672, Romania

*Corresponding author: C.Ș. Novac, e-mail: cristiana.novac@usamvcluj.ro

\section{RESEARCH ARTICLE}

\begin{abstract}
Bovine mastitis is a cause of major financial losses in the dairy industry worldwide. Bovine mastitis is defined as the inflammatory response of the udder tissue due to either physical trauma or infections caused by different microorganisms, especially bacteria. More than 135 pathogenic agents have been identified from bovine mastitis. The aim of the study was to identify the bacterial genera involved in mastitis etiology in cattle and to test the antimicrobial susceptibility to antibiotics. The samples were collected before morning milking and evaluated by microscopic and cultural examination, and further identified using biochemical methods. The evaluation of antimicrobial susceptibility was performed using Kirby-Bauer disk diffusion test. Overall, Staphylococcus spp., Bacillus spp., Streptococcus spp. Micrococcus spp., Escherichia spp. and Trueperella spp. were identified. The most frequent bacterial associations were observed for Staphylococcus-Bacillus, Bacillus-Streptococcus and Staphylococcus-Micrococcus. Regarding the antimicrobial sensitivity profile, the majority of the bacterial strains we have isolated were classified as sensitive, with the exception of Escherichia coli, with one strain being resistant to all tested antibiotics. Among the antimicrobials, the Amoxicillin and clavulanic acid was the most efficient one and Trimethoprim/Sulfamethoxazole did not exhibit an inhibitory activity in 19 milk samples.
\end{abstract}

Keywords: cow milk; bovine mastitis; AMR, antimicrobial susceptibility.

Received: 28 April 2021

Accepted: 06 May 2021

Published: 14 May2021

DOI:

10.15835/buasvmcn-vm:2021.0005

(c) $(1) \Theta \Theta(2021$ Authors. The papers published in this journal are licensed under the Creative Commons AttributionNonCommercial-NoDerivatives 4.0 International

License

\section{INTRODUCTION}

Mastitis is a cause of major financial losses in the dairy industry worldwide. These losses are mainly due to reduced yield and poor milk quality caused by changes in milk composition (Botrel et al., 2010, Cheng and Han, 2020). The significant economic impact translates into decreased milk production, discarded milk and pre-term drying off. Moreover, cow mastitis is also associated with premature culling and even death (Gomes and Henriquez, 2016). Bovine mastitis is defined as the inflammatory response of the udder tissue due to either physical trauma or infections caused by different microorganisms, especially bacteria (Cheng and Han, 2020). More than 135 pathogenic agents have been identified from bovine mastitis (Abdi et al., 2021). Bacteria that are commonly involved in the etiology of cow mastitis are classified as either contagious or environmental pathogens. Contagious pathogens are transmitted from cow to cow most often during milking time, whereas the second category of bacteria have their reservoir in the animals' environment, in the bedding and housing of the herd (Royster and Wagner, 2015). Major contagious mastitis pathogens are represented by Staphylococcus aureus, Streptococcus agalactiae and Mycoplasma spp. On the other hand, the environmental pathogens group includes a wide range of microorganisms, such as coliforms (E. coli, Enterobacter spp., Klebsiella spp., Citrobacter spp.), Trueperella pyogenes, non-aureus staphylococci (NAS), streptococci (Streptococcus dysagalactiae, Streptococcus uberis) and other bacteria such as Pseudomonas spp., Proteus spp., Aerococccus spp., yeasts or algae (Prototheca spp.) (Abdi et al., 2021). 
Unlike contagious pathogens, environmental ones do not usually live on the cow's udder and teat skin, thus they are considered opportunistic microorganisms (Cameron et al., 2016).

Based on the degree of inflammation, bovine mastitis can be classified into 3 major groups: clinical, subclinical and chronic mastitis. Clinical mastitis evolves with visible signs and abnormal milk secretion, whereas subclinical mastitis shows no visible changes in the udder or milk (Barlow et al., 2011). In this case, milk production is often reduced and the milk somatic cell count is significantly increased. Conversely, chronic mastitis is defined as a long term inflammatory process that can last for several months. Nevertheless, the subclinical form accounts for more financial losses compared to clinical mastitis, due to the fact that it is quite difficult to quantify because it evolves with no visible signs and the diagnosis requires further milk testing (Cheng and Han, 2020). The most frequently isolated pathogens from clinical cases of mastitis are Escherichia coli, Streptococcus uberis and Staphylococcus aureus, whereas subclinical mastitis is most often caused by coagulase-positive staphylococci (CPS), NAS and streptococci. E. coli is less frequently involved in the etiology of subclinical forms (Persson Waller et al., 2011). However, these four major groups (coliforms, CPS, NAS, streptococci) represent a real concern for the public health, as they can play an important role as reservoir for antimicrobial resistance genes and, moreover, they can be involved in spreading the genes to other pathogenic and commensal microorganisms in the farm (Boireau et al., 2018).

Because dairy cattle mastitis is mainly caused by bacteria, antibiotics are an important component of the therapeutic approach and control programs (Boireau et al., 2018). Thus, intramammary infections are the most common cause for antimicrobial use in lactating cows. Due to this excessive use of antibiotics, resistant bacterial strains have been isolated from bovine mastitis in the last few years (Alekish et al., 2013). Surveillance of the antimicrobial resistance is extremely important in order to minimize the risk for selection of resistant bacterial strains and also limit the spread of antimicrobial resistance which poses a serious threat for the public health system, making this problem a global concern (Persson Waller et al., 2011). In recent years, there has been observed a growing interest in the emergence and diffusion of antibiotic resistance that results from the aggressive use of antimicrobials in the veterinary sector.

The phenomenon of antimicrobial resistance associated with bovine mastitis pathogens has two relevant aspects. The first one is related to a low cure rate after the treatment of clinical cases and the second one is the potential transmission of resistant microorganisms to humans via the food chain (Sol et al., 2000; Pinzón-Sánchez and Ruegg, 2011). Nevertheless, this transmission is possible whenever subclinical mastitis is involved, due to the presence of resistant strains in bulk tank milk that may be passed to humans via raw milk (Tenhagen et al., 2006). The literature is abundant in studies focused on antimicrobial resistant bacteria isolated from bovine mastitis (De Oliveira et al., 2000; Tenhagen et al., 2006; Pitkala et al., 2008; Oliver and Murinda, 2012; Liu et al., 2014; Thomas et al., 2015).

In this context, the aim of this paper is to identify the bacterial genera involved in mastitis etiology in cattle and to test the antimicrobial susceptibility to antibiotics.

\section{MATERIALS AND METHODS}

A total number of twenty-one milk samples from cattle diagnosed with clinical mastitis collected from three farms in Alba County have been included in the study. All samples were collected before morning milking, after teats end cleaning with $70 \%$ ethanol. First streams of foremilk were discarded and milk was collected aseptically in sterile plastic containers. Specimens were processed the same day, refrigerated during transportation and had modified consistency, aspect and colour. Milk samples have been initially evaluated by microscopic and cultural examination, and further identified using biochemical methods. Microscopic examination involved stained smears using Gram staining technique, and offered details regarding the shape, size and arrangement, being considered useful for orienting the next step, cultural examination.

The culture media used were represented by blood and Sabouraud agar. In this concern, a loopful of milk was used to inoculate blood agar medium and Sabouraud agar. Blood agar plates were incubated at $37^{\circ} \mathrm{C}$ for $18-24 \mathrm{~h}$, while Sabouraud agar plates at $30^{\circ} \mathrm{C}$ for $48-72 \mathrm{~h}$. The cultural examination is conferring details regarding the size, shape and colour of the colonies. The type of hemolysis (alpha, beta, gamma) was determined on blood agar plates and the identification of bacteria was performed by evaluating biochemical characters (catalase, oxidase and hyaluronidase). Therefore, a catalase test was conducted on Gram-positive cocci to differentiate staphylococci from streptococci and the oxidase test was utilized to differentiate the Enterobacteriaceae from Gram-negative non-Enterobacteriaceae bacteria. Oxidase negative microorganisms were further inoculated on MacConkey agar for the evaluation of lactose fermentation. Milk samples with two different colonies were considered a mixed infection.

The evaluation of antimicrobial susceptibility was performed using Kirby-Bauer disk diffusion test. In this concern, Mueller-Hinton agar plates have been flooded with $1 \mathrm{ml}$ from the suspension of mixed flora isolated colonies in broth, at the density of 0.5 on McFarland scale, using the electronic density evaluator Densicheck ${ }^{\mathrm{TM}}$ Plus (Biomérieux). The antibiotics were represented by amoxicillin and clavulanic acid (AMC) 20/10 $\mu$ g, cefotaxime (CTX) 30 $\mu$ g, doxycycline

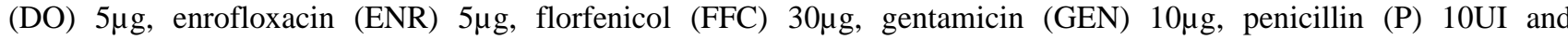
trimethoprim/sulfamethoxazole (STX) $25 \mu \mathrm{g}$. Breakpoints for zone diameter in mm were considered as follows: AMC 22, CTX 20, DO 22, ENR 18, FFC 17, GEN 21, P 26 and STX 22. 


\section{RESULTS AND DISCUSSIONS}

Bacteria were identified in twenty $(95.23 \%)$ out of twenty-one milk samples evaluated. From a total of nine milk samples a single bacterial genus was identified, whereas in the remaining eleven milk samples, bacterial associations were present. Individual bacteria identified in mik samples were represented by Staphylococcus $\mathrm{spp} .(\mathrm{n}=4)$, Bacillus spp. $(\mathrm{n}=$ 2), E. coli $(\mathrm{n}=2)$ and Trueperella spp. $(\mathrm{n}=1)$. Overall, Staphylococcus spp. was identified in a total of nine samples, Bacillus spp. in 8, Streptococcus spp. in 5, Micrococcus spp. in 3, while E. coli and Trueperella spp. each in 2 samples. All samples were negative for yeast growth on Sabouraud agar. The results regarding the bacterial associations are presented in Table 1.

Table 1. The bacterial associations identified in milk samples

\begin{tabular}{lc}
\hline \multicolumn{1}{c}{ Bacterial associations } & Number of milk samples \\
\hline Staphylococcus, Bacillus & 3 \\
\hline Bacillus, Streptococcus & 3 \\
\hline Staphylococcus, Micrococcus & 2 \\
\hline Staphylococcus, Trueperella & 1 \\
\hline Staphylococcus, Streptococcus & 1 \\
\hline Streptococcus, Micrococcus & 1 \\
\hline
\end{tabular}

For the antimicrobial susceptibility testing, the overall results revealed that for the majority of milk samples included in the study, at least one antibiotic was efficient and recommended. The most efficient antimicrobial was represented by $\mathrm{AMC}$, as only seven bacterial isolates from five samples were resistant. AMC was also recommended for the treatment of eight cattle, the best in this concern. P and ENR also demonstrated good overall results, each of these antibiotics being recommended to be used in order to treat four cows. GEN was recommended in the treatment of two cattle, while DO for one.

Table 2. The number of resistant bacterial strains for each tested antibiotic

Staphylococcus spp. $\quad$ Streptococcus spp. Bacillus spp. Micrococcus spp. Trueperella spp. E. coli Total

\begin{tabular}{cccccccc}
\hline AMC & 2 & 1 & 2 & 1 & 0 & 1 & 7 \\
\hline CTX & 1 & 2 & 1 & 2 & 0 & 2 & 8 \\
\hline DOX & 2 & 1 & 2 & 1 & 1 & 0 & 2 \\
\hline ENR & 1 & 1 & 1 & 1 & 0 & 2 \\
\hline FFC & 1 & 0 & 1 & 1 & 0 & 1 & 4 \\
\hline GEN & 1 & 2 & 7 & 3 & 0 & 2 & 12 \\
\hline P & 4 & 5 & 18 & 10 & 2 & 2 & 27 \\
\hline STX & 8 & 13 & & 7 & 2 & 13 \\
\hline Total & 20 & & & & 1 & 0 & 1 \\
\hline
\end{tabular}

From the total of twenty milk samples evaluated for antimicrobial susceptibility testing, six bacterial isolates were resistant to GEN, seven to AMC and FFC, seven to ENR, eight to CTX, ten to DO and 19 to STX. Regarding multidrug resistance (MDR), the strains were so considered if the resistance was recorded for at least three antimicrobial substances from three different classes (Magiorakos et al., 2012). The only MDR strain was E. coli, with total resistance for all tested antibiotics for one strain, whereas the second isolated strains showed resistance to five out of eight antimicrobials. Thus, CTX, DOX, ENR, P and STX had no efficiency against this strain of E. coli. Furthermore, from all antibiotics included in the study, STX showed moderate inhibitory activity in case of one strain from the total of twenty evaluated and no activity for all the other. The results regarding the resistance to antimicrobials for each genus are presented in Table 2.

Therefore, from a total of twenty positive milk samples, we have isolated 6 bacterial genera, as follows: Staphylococcus spp., Bacillus spp., Streptococcus spp., Micrococcus spp., Escherichia spp. and Trueperella spp. The results of our study are consistent with other papers in the literature focused on bovine mastitis (Tenhagen et al., 2006, Persson et al., 2011). Although the prevalence of udder pathogens may vary between countries, studies generally describe staphylococci, streptococci and E. coli as the major pathogenic agents involved in the etiology of clinical mastitis (Botrel et al., 2010; Thomas et al., 2015). However, in recent years, farmers have started to implement different measures in order to prevent intramammary infections, such as milk hygiene, better farm management and an appropriate use of antimicrobials. Such measures tend to reduce the incidence of mastitis. Moreover, a change in the hierarchy of mastitis pathogens can be observed. For example, Staphyloccocus aureus and Streptococcus agalactiae seem to have a lower prevalence nowadays, but on the other hand, CNS and Trueperella spp. are more frequently isolated from mastitis milk samples (Zhang et al., 2018). However, in our study the most encountered bacterial genus was Staphylococcus spp., followed by Bacillus spp. 
and Streptococcus spp., showing that both staphylococci and streptococci are still major causative agents of clinical bovine mastitis in Romania, our results being in accordance with other studies which obtained a similar prevalence for Staphylococcus spp., Streptococcus spp. and E. coli (Tenhagen et al., 2006; Persson et al., 2011). Regarding other Grampositive bacteria, a study conducted by Tenhagen et al. (2006) focused on the prevalence of mastitis pathogens in dairy cows from Germany, revealed a higher prevalence of Trueperella spp. compared to the results of the present research. On the other hand, the same authors isolated Enterococcus faecalis and Enterococcus faecium, but with a much lower frequency, $0.2 \%$ compared to other strains (Tenhagen et al., 2006). Abdi et al. (2021), in a study conducted on both clinical and subclinical mastitis in the USA, showed that the most frequently isolated pathogens from cases of clinical mastitis were E. coli, Klebsiella oxytoca and Streptococcus dysagalactiae, with a significantly higher percentage of E. coli strains (79.4\% of all E. coli were isolated from clinical mastitis) compared to the results of our study. Conversely, the same study revealed that the main microorganisms involved in the etiology of subclinical intramammary infections were represented by Staphylococcus aureus, Klebsiella pneumoniae and Streptococcus uberis (Abdi et al., 2021). However, in contrast with the results obtained in the above mentioned studies, the present paper also revealed the presence of Bacillus spp., with a significantly high prevalence regarding the milk samples included in the study (8 out of 20 positive samples).

Nowadays, antimicrobial therapy is commonly used for both mastitis prevention and control, but unfortunately, treatments failures are encountered quite often, due to the emergence of antimicrobial resistant bacterial strains (Saini et al., 2012). This resistance of mastitis pathogens to commonly used antibiotics is a well-documented problem in the industry of dairy cows and it is now being closely monitored (Tenhagen et al., 2006). Moreover, identifying the bacterial strains involved in the etiology of bovine mastitis, as well as their sensitivity to antimicrobials at the level of region or country is extremely important in order to preserve and adapt the therapeutic protocols (Botrel et al., 2010). Nevertheless, another serious threat which is closely related to the aggressive use of antibiotic in the treatment of cattle mastitis is the potential risk of transmission of resistant pathogens to humans via raw milk and raw milk products, leading to a possible public health hazard (Waller et al., 2011).

Overall, the prevalence of antimicrobial resistance was low. The most resistant bacterium isolated from the milk samples we have analyzed was represented by $E$ coli. Therefore, out of the two isolated strains, one was resistant to all tested antibiotics. The only efficient antimicrobials against this microorganism were AMC, FFC and GEN, with an inhibition zone diameter of $22.49 \mathrm{~mm}, 22.87 \mathrm{~mm}$ and $22.26 \mathrm{~mm}$ respectively. The results of our study are consistent with other studies in the literature regarding coliform mastitis and the antibiotic resistance patterns (Thomas et al., 2015, Abdi et al., 2021). The antimicrobial resistance of $E$. coli has been frequently reported in recent years, especially regarding bacterial strains isolated from cases of mastitis in dairy ruminants (Liu et al., 2014; Zhang et al., 2018). The high levels of antimicrobial resistance for this strain in particular could be explained by long-term use of these antibiotics in dairy herds, without a prior bacteriological culture of milk samples and a sensitivity test. Throughout the world, E. coli is one of the most important pathogens associated with cow clinical mastitis and even though it is considered an envinronmental agent, it is responsible for causing serious tissue damage. Moreover, the antibiotic resistance developed by this microorganism is one of the major problems whenever recommending a treatment, due to low cure rates (Liu et al., 2014).

Regarding Gram-positive cocci sensitivity results, the present study revealed a low prevalence of antibiotic resistant strains. However, the isolated strains belonging to Staphylococcus and Streptococcus genus showed a rather high resistance to P and STX, the results being in accordance with other papers (Abdi et al., 2021). The same observation is available for Bacillus spp. On the other hand, the most susceptible bacteria to antimicrobials was Trueperella spp., the strains being resistant only to STX. Overall, AMC, a frequently used antimicrobial agent, showed a good activity against both Grampositive and Gram-negative results. However, in order to avoid the spread of antibiotic resistant bacterial strains, it is highly recommended to perform microbiological tests on milk samples, to identify the pathogens and perform the antibiotic sensitivity testing.

\section{CONCLUSIONS}

Overall, from a total of 21 milk samples collected from cows diagnosed with clinical mastitis, 20 samples were positive for bacterial growth. Thus, bacteria belonging to 6 different genera were isolated. Staphylococcus spp. was the most frequently isolated genus, followed by Bacillus spp. and Streptococcus spp. Other Gram-positive bacteria were represented by Micrococcus spp. and Trueperella spp., which have been identified in a small number of samples. Among the Gramnegative microorganisms, we have isolated $E$. coli, but with a low frequency. Regarding the antimicrobial sensitivity profile, the majority of the bacterial strains we have isolated were classified as sensitive, with the exception of $E$. coli, with one strain being resistant to all tested antibiotics. Among the antimicrobials, the AMC was the most efficient one and STX did not exhibit an inhibitory activity in 19 out of 20 milk samples.

Author Contributions: G.C.N. Conceived and designed the analysis; C.S.N. Collected the data; C.M.B. Contributed data or analysis tools; I.A.M. Performed the analysis; N.I.F. Provided the resources; G.C.N., C.S.N. Wrote the paper.

\section{Acknowledgments}

This research did not receive any specific grant from funding agencies in the public, commercial, or not-for-profit sectors. 


\section{Conflicts of Interest}

The authors declare that they do not have any conflict of interest.

\section{REFERENCES}

1. Abdi RD, Gillespie BE, Ivey S, Pighetti GM, Almeida RA, Kerro Dego O. Antimicrobial Resistance of Major Bacterial Pathogens from Dairy Cows with High Somatic Cell Count and Clinical Mastitis. Animals. 2021 Jan;11(1):131.

2. Alekish MO, Al-Qudah KM, Al-Saleh A. Prevalence of antimicrobial resistance among bacterial pathogens isolated from bovine mastitis in northern Jordan. Rev. Med. Vet. 2013 Jun 1;164(6):319-26.

3. Barlow J. Mastitis therapy and antimicrobial susceptibility: a multispecies review with a focus on antibiotic treatment of mastitis in dairy cattle. Journal of mammary gland biology and neoplasia. 2011 Dec 1;16(4):383-407.

4. Boireau C, Cazeau G, Jarrige N, Calavas D, Madec JY, Leblond A, Haenni M, Gay É. Antimicrobial resistance in bacteria isolated from mastitis in dairy cattle in France, 2006-2016. Journal of dairy science. 2018 Oct 1;101(10):9451-62.

5. Botrel MA, Haenni M, Morignat E, Sulpice P, Madec JY, Calavas D. Distribution and antimicrobial resistance of clinical and subclinical mastitis pathogens in dairy cows in Rhône-Alpes, France. Foodborne pathogens and disease. 2010 May 1;7(5):479-87.

6. Cameron M, Saab M, Heider L, McClure J, Rodriguez-Lecompte JC, Sanchez J. Antimicrobial susceptibility patterns of environmental streptococci recovered from bovine milk samples in the Maritime provinces of Canada. Frontiers in veterinary science. 2016 Sep 15;3:79.

7. Cheng WN, Han SG. Bovine mastitis: risk factors, therapeutic strategies, and alternative treatments-A review. AsianAustralasian Journal of Animal Sciences. 2020 Nov;33(11):1699.

8. De Oliveira AP, Watts JL, Salmon SA, Aarestrup FM. Antimicrobial susceptibility of Staphylococcus aureus isolated from bovine mastitis in Europe and the United States. Journal of dairy science. 2000 Apr 1;83(4):855-62.

9. Gomes F, Henriques M. Control of bovine mastitis: old and recent therapeutic approaches. Current microbiology. 2016 Apr;72(4):377-82.

10. Liu Y, Liu G, Liu W, Liu Y, Ali T, Chen W, Yin J, Han B. Phylogenetic group, virulence factors and antimicrobial resistance of Escherichia coli associated with bovine mastitis. Research in microbiology. 2014 May 1;165(4):273-7.

11. Magiorakos AP, Srinivasan A, Carey RT, Carmeli Y, Falagas MT, Giske CT, Harbarth S, Hindler JT, Kahlmeter G, Olsson-Liljequist B, Paterson DT. Multidrug-resistant, extensively drug-resistant and pandrug-resistant bacteria: an international expert proposal for interim standard definitions for acquired resistance. Clinical microbiology and infection. 2012 Mar 1;18(3):268-81

12. Oliver SP, Murinda SE. Antimicrobial resistance of mastitis pathogens. Veterinary Clinics: Food Animal Practice. 2012 Jul 1;28(2):165-85.

13. Persson Y, Nyman AK, Grönlund-Andersson U. Etiology and antimicrobial susceptibility of udder pathogens from cases of subclinical mastitis in dairy cows in Sweden. Acta Veterinaria Scandinavica. 2011 Dec;53(1):1-8.

14. Pinzón-Sánchez C, Ruegg PL. Risk factors associated with short-term post-treatment outcomes of clinical mastitis. Journal of dairy science. 2011 Jul 1;94(7):3397-410.

15. Pitkälä A, Koort J, Björkroth J. Identification and antimicrobial resistance of Streptococcus uberis and Streptococcus parauberis isolated from bovine milk samples. Journal of dairy science. 2008 Oct 1;91(10):4075-81.

16. Royster E, Wagner S. Treatment of mastitis in cattle. Veterinary Clinics: Food Animal Practice. 2015 Mar 1;31(1):17-46.

17. Saini V, McClure JT, Léger D, Keefe GP, Scholl DT, Morck DW, Barkema HW. Antimicrobial resistance profiles of common mastitis pathogens on Canadian dairy farms. Journal of dairy science. 2012 Aug 1;95(8):4319-32.

18. Sol J, Sampimon OC, Barkema HW, Schukken YH. Factors associated with cure after therapy of clinical mastitis caused by Staphylococcus aureus. Journal of dairy science. $2000 \mathrm{Feb}$ 1;83(2):278-84.

19. Tenhagen BA, Köster G, Wallmann J, Heuwieser W. Prevalence of mastitis pathogens and their resistance against antimicrobial agents in dairy cows in Brandenburg, Germany. Journal of Dairy Science. 2006 Jul 1;89(7):2542-51.

20. Thomas V, de Jong A, Moyaert H, Simjee S, El Garch F, Morrissey I, Marion H, Vallé M. Antimicrobial susceptibility monitoring of mastitis pathogens isolated from acute cases of clinical mastitis in dairy cows across Europe: VetPath results. International journal of antimicrobial agents. 2015 Jul 1;46(1):13-20.

21. Waller KP, Aspán A, Nyman A, Persson Y, Andersson UG. CNS species and antimicrobial resistance in clinical and subclinical bovine mastitis. Veterinary microbiology. 2011 Aug 26;152(1-2):112-6.

22. Zhang D, Zhang Z, Huang C, Gao X, Wang Z, Liu Y, Tian C, Hong W, Niu S, Liu M. The phylogenetic group, antimicrobial susceptibility, and virulence genes of Escherichia coli from clinical bovine mastitis. Journal of dairy science. 2018 Jan 1;101(1):572-80. 